\title{
Comparison of Diabetes-Dependent Quality of Life (ADDQoL) in Patients with T2DM in Poland, The Czech Republic, and Slovakia
}

This article was published in the following Dove Press journal: Diabetes, Metabolic Syndrome and Obesity: Targets and Therapy

\author{
Sylwia Krzemińska (D) \\ Ewelina Bąk (D) ${ }^{2}$ \\ Lenka Šáteková $\mathbb{D D}^{3}$ \\ Andrea Polanská ${ }^{4}$ \\ Kateřina Hašová ${ }^{4}$ \\ Milan Laurinc $\mathbb{D}^{5}$ \\ 'Department of Clinical Nursing, Faculty \\ of Health Science, Wroclaw Medical \\ University, Wroclaw, Poland; ${ }^{2}$ Faculty of \\ Health Sciences, University of Bielsko- \\ Biala, Bielsko-Biala, Poland; ${ }^{3}$ Department \\ of Nursing, Faculty of Health Sciences, \\ Palacký University Olomouc, Olomouc, \\ Czech Republic; ${ }^{4}$ University Hospital \\ Ostrava, Ostrava-Poruba, Czech \\ Republic; ${ }^{5}$ National Institute of \\ Cardiovascular Children's Cardiology \\ Center, Bratislava, Slovakia
}

Introduction: The purpose of the paper was to perform a comparative analysis of the impact of T2DM on QoL, including specific ADDQoL domains and associations between QoL, selected socio-demographic factors (including gender, age, education, residence, marital status, professional activity) or clinical parameters (HbAlc levels, fasting blood glucose, BMI, duration of DM, complications, treatment used), in adult diabetic patients from Poland, the Czech Republic and Slovakia. The study group included 608 patients diagnosed with T2DM. There were 214 patients from Poland, 196 from the Czech Republic and 198 from Slovakia. Results: Overall, respondents from all three countries rated their QoL as good or very good. The mean scores for the item "If I did not have diabetes, my quality of life would be" were slightly higher for Poles than for Slovaks and Czechs. In the ADDQoL results, the weighted impact scores were negative for all domains. The lowest scores in all three countries were found for "freedom to eat, for all patients; the highest for "living conditions". For Polish patients, the linear regression model demonstrated the following significant AWI predictors: pre-university education and past smoking. For Czech patients, the linear regression model demonstrated that none of the characteristics analyzed were significant independent predictors of AWI. In the For Slovak patients, the linear regression model demonstrated the following significant AWI predictors: higher education and concurrent heart failure.

Conclusion: In summary, our findings demonstrate that T2DM has a negative impact on all aspects of patients' QoL, which is the strongest in terms of the freedom to eat and dietary habits, regardless of the country. Education, past smoking, and concurrent heart failure were the only independent predictors of QoL in our study. This suggests a need for further research that would include more variables and a larger number of patients.

Keywords: diabetes mellitus, quality of life

Global epidemiological data indicate a rapid increase in the incidence of diabetes mellitus (DM). Currently, DM is among the most common causes of death worldwide. The condition is a serious public health concern. According to the literature data, there were 463 million patients with DM in 2019, and by 2045 this number is expected to have grown to 700 million. ${ }^{1}$ DM prevalence differs between continents, depending on the presence of risk factors and some genetic differences associated with its incidence. Until recently, type 2 diabetes mellitus (T2DM) was considered a condition characteristic for residents of highly developed and wealthy countries, although at present the number of T2DM cases is also growing in developing countries. $^{2}$
Correspondence: Sylwia Krzemińska Department of Clinical Nursing, Faculty of Health Sciences, Wroclaw Medical University, Bartla 5, Wroclaw 5I-6I8, Poland

Email sylwia.krzeminska@umed.wroc.pl
Diabetes, Metabolic Syndrome and Obesity: Targets and Therapy 2020:13 3773-3786 
The WHO defines quality of life (QoL) as an individual's perception of their position in life in the context of the culture and value systems in which they live, and in relation to their goals, expectations, standards, and concerns. Therefore, it is a broad concept, involving complex relationships between physical health, psychological status, personal beliefs, social relationships, and many associated characteristics of one's environment. ${ }^{3}$

Traditionally, the impact of chronic illness had been measured by morbidity and mortality. However, researchers have begun to appreciate health-related quality of life (HRQoL) as a valid and important outcome of medical interventions. In diabetic patients, QoL measurement can predict an individual's capacity for dealing with the illness and maintaining long-term health and wellbeing. ${ }^{4}$

Long-term T2DM management usually requires diet and lifestyle changes and strict adherence to treatment, all of which may affect QoL. When QoL and/or satisfaction with treatment are disturbed by the therapeutic regime, this can adversely affect the patient's perception of their QoL and increase the risk of complications. Insulin therapy and the associated complications are also linked to poorer QoL. ${ }^{5,6}$ A literature review shows that DM has a negative impact on the patient's physical and psychological wellbeing, as well as their social functioning, and thus leads to a deterioration in QoL both in patients with $\mathrm{T}_{1 \mathrm{DM}^{7,8}}$ and in those with T2DM. ${ }^{9-13}$

As few papers exist that compare QoL between neighboring countries, the authors decided to investigate the issue in three neighboring European countries: Poland, the Czech Republic, and Slovakia.

Based on data provided by the Polish National Health Fund and the Diabetes-Coalition organization, there are about 3.5 million people with DM in Poland, which constitutes $9 \%$ of the entire population. T2DM has been diagnosed in 2 million people, or $6 \%$ of the population. Considering that $6 \%$ of the Polish population has T2DM, ${ }^{14}$ with a maximum error of $3 \%$ and a confidence level of $90 \%$, the minimum sample size can be estimated at 163 patients. For the Czech Republic, with a population of $10,650,000$, a structure ratio of $7.38 \%,{ }^{15}$ confidence of $95 \%$, and an error of estimation of $4 \%$, the minimum size would be 164 patients. And for Slovakia, with a population of $5,450,000$, a structure ratio of $5.85 \%,{ }^{16}$ confidence of $95 \%$, and an error of estimation of $4 \%$, the minimum size would be 132 patients.

Ultimately, 214 people from Poland, 196 from the Czech Republic and 198 from Slovakia were qualified for the research after excluding incorrectly completed questionnaires. Therefore, our sample size was sufficient for analysis.

\section{Purpose}

The purpose of the paper was to perform a comparative analysis of the impact of T2DM on QoL, including specific ADDQoL domains and associations between QoL, selected socio-demographic factors (including gender, age, education, residence, marital status, professional activity) or clinical parameters (HbA1c levels, fasting blood glucose, BMI, duration of DM, complications, treatment used), in adult diabetic patients from Poland, the Czech Republic and Slovakia.

The study was approved by the Bioethics Committee of the Beskidy Regional Chamber of Physicians in BielskoBiała, Poland, on 11 February 2016 (approval no. 2016/02/ 11/1), and the Bioethics Committee of the Wrocław Medical University (no. 621/2017). All participants were informed about the content of the study and gave their informed consent to participate in it. The study protocol was prepared in accordance with the Helsinki Declaration.

\section{Material}

A total of 660 patients were examined, 608 remained after excluding incorrectly completed questionnaires.

There were 214 patients from Poland (out of 240 examined), including 100 women and 114 men aged $61.53 \pm 7.76$ years; 196 from the Czech Republic (out of 210 examined), including 82 women and 114 men aged $59.16 \pm 7$ years; and 198 from Slovakia (out of 210 examined), including 96 women and 102 men aged 58.8 57.02 years.

All participants had been diagnosed with T2DM. In Poland, the study was performed at the Diabetes Clinic of the Bielsko-Biała Regional Hospital, the Diabetes Clinic of the Medi-Diab Nonpublic Medical Center in Katowice, and the Kosmonautów Health Center in Wrocław. In the Czech Republic, the location was the Ostrava University Hospital, and in Slovakia, the Svet Zdrovia hospital network, with the consent of the management of the institutions. The study was performed between March 2016 and August 2019.

Patients with T2DM were treated with a diet and oral antidiabetic drugs, with insulin if necessary. All patients reported for follow-up at the diabetes clinic approx once every three months. During their appointments at the clinic, the patients had their glucose and HbAlc levels 
measured, their further pharmaceutical treatment was planned, and they underwent education focused on the appropriate diet and food content, considering levels of carbohydrates, protein and fat. Aerobic physical training was also recommended for the patients.

\section{Methods}

The values of the glucose measures (fasting blood glucose and glycated hemoglobin-HbAlc levels) were determined in all the patients. Next, surveys were carried out, using the following instruments: a demographic and clinical survey, and the ADDQoL questionnaire. The survey also collected the personal data of each patient: age, gender, place of residence, education, marital status, professional activity, body weight, comorbidities, duration of DM, DM complications, and medications taken. Only diabetes complications and comorbidities confirmed by a specialist were considered.

The ADDQoL questionnaire (developed by Clare Bradley) is a diabetes-specific instrument used for evaluating QoL both in T1DM and T2DM patients. ${ }^{17}$ It consists of two general questions referring to the patient's QoL: 1) the current overall level of QoL, measured on a 7-grade scale (excellent, very good, good, neither good nor bad, bad, very bad, and extremely bad); 2) the specific influence of DM on QoL, measured on a 5-grade scale (very much better, much better, a little better, the same, and worse). The remaining components refer to 19 domains of QoL without the disease, and to the influence of DM on these aspects of life. Each domain includes two components: impact (from -3 , maximum negative impact of DM, to +1 , positive impact of $\mathrm{DM}$ ), and importance (3-very important, 0 - not at all important). The product of impact and importance ratings determines the weighted impact (WI) score. This value may range from -9 to +3 for every domain of the ADDQoL examined. The lower the WI score, the worse the aspect of life within the scope of a given domain. The average value of the weighted impact (AWI) score is also calculated for the whole scale. The AWI score is derived by dividing the sum of the weighted ratings by the number of applicable domains. The ADDQoL comprises the following domains: leisure activities, working life, journeys, holidays, physical health, family life, friendship and social life, personal relationships, sex life, physical appearance, self-confidence, motivation, people's reactions, feelings about the future, financial situation, living conditions, dependence on others, freedom to eat, and freedom to drink. ${ }^{17,18}$ The
ADDQoL was used in our study with the consent and license from the author, Clare Bradley (Health Psychology Research Unit, Royal Holloway, University of London) via www.healthpsychologyresearch.com. The license number for the Polish language version was CB521. The study in Poland relied on the Polish language version of the ADDQoL, as its psychometric properties, determined earlier, indicate that it is a reliable tool for the assessment of QoL in adult Polish patients with T1DM or T2DM. ${ }^{19}$

The other parts of the study relied on the Czech and Slovak versions of the ADDQoL. The license number for the Czech language version was CB636, and for the Slovak version it was CB638. Validation in the respective countries also confirmed the utility of the instrument for the evaluation of patients with T2DM in the Czech Republic and Slovakia. ${ }^{20,21}$

Before the start of the study, each patient was informed about its purpose by the authors. The questionnaires were completed personally and anonymously by each patient during an appointment with a physician. The time needed for survey completion was 20-30 minutes.

\section{Statistical Analysis}

Comparisons of qualitative variables in groups were performed using the chi-squared test (with Yates' correction for $2 \times 2$ tables) or Fisher's exact test (for low expected values). Comparisons of quantitative variables in two groups were performed using the Mann-Whitney test. Comparisons of quantitative variables in more than two groups were performed using the Kruskal-Wallis test. The Dunn test was used for post hoc analysis. Multivariate analysis of the simultaneous impact of many independent variables on one quantitative dependent variable was performed by linear regression. 95\% confidence intervals were reported along with regression parameters. These analyses were performed with a 0.05 significance threshold. The R software, version 3.6.2 was used. ${ }^{22}$

\section{Results}

Statistically significant differences were also found when the data were compared between the countries. Polish patients were older $(61.53 \pm 7.76$ years $)$ than Czechs (59.16 \pm 7$)$ and Slovaks (58.8 \pm 7.02$)$. BMI was significantly higher among Czech (26.87 \pm 3.79$)$ and Polish respondents (26.18 \pm 4.67$)$ than in the Slovak group (25.28 \pm 2.6$)$. The duration of T2DM was longer among Slovaks than among Czechs $(6 \pm 2.25$ vs $5.36 \pm 2.4, \mathrm{p}=0.02)$. The number of 
patients who had completed higher education was the largest in the Polish group (16.36\%). Poles were most likely and Slovaks least likely to live in cities $(64.95 \%$ vs $37.88 \%, \mathrm{p}<0.001)$. Professional activity was the highest among Czechs and the lowest among Poles $(48.98 \%$ vs $29.91 \%, \mathrm{p}<0.001)$. Poles were most likely to smoke $(36 \%)$ and drink alcohol (35.51\%), while Slovaks were the least likely (17.17\%). Regarding comorbidities, the rate of hypertension was the highest among Slovaks (96.46\%) and the lowest among Poles (80.37\%). Concerning DM complications, Slovaks had the highest rate of retinopathy $(55.05 \%)$ and the lowest rate of nephropathy (5.56\%), while the opposite was observed in the Polish group (Table 1).

\section{ADDQoL by Country}

The mean overall QoL scores were slightly higher among Slovaks (3.47 \pm 0.98$)$ than among Poles $(3.41 \pm 0.92)$ and Czechs $(3.38 \pm 0.93)$. The mean scores for the item "If I did not have diabetes, my quality of life would be" were slightly higher for Poles (2.65 \pm 0.92$)$ than for

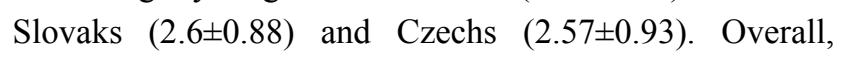
respondents from all three countries rated their QoL as good or very good: Slovaks-59.19\%, Poles-58.41\%, Czechs-57.57\%. Only one Polish respondent reported "excellent" QoL. In response to the item "If I did not have diabetes, my quality of life would be" all three groups expected it to be better: Slovaks-84.84\%, Czechs-82.65\%, Poles-81.31\%. Again, only one Polish respondent expected their QoL would be worse. No significant differences were found (all $\mathrm{p}>0.05$ ) (Table 2).

Table 3 shows weighted impact scores for each country. In the study group, the weighted impact scores were negative for all domains. The lowest scores in all three countries were found for "freedom to eat": $-4.23 \pm 2.6$ for Poland, $-4.19 \pm 2.44$ for Slovakia, and $-4.16 \pm 2.49$ for the Czech Republic; the highest-for "living conditions": $1.22 \pm 1.99$ for Poland, $-1.29 \pm 2.03$ for the Czech Republic, and $-1.31 \pm 2.02$ for Slovakia (Table 4). Negative WI scores in the analyzed domains ranged between -1.22 ("living conditions") and -4.23 ("freedom to eat") for Polish patients, from -1.29 ("living conditions") to -4.16 ("freedom to eat") for Czechs, and from -1.31 ("living conditions") to -4.19 ("freedom to eat") for Slovaks (Table 4). No correlations were statistically significant (all $\mathrm{p}>0.05$ ). The average weighted impact (AWI) score in the group was lowest for Slovakia $(-2.38 \pm 1.64)$, followed by Poland
$(-2.31 \pm 1.6)$ and the Czech Republic $(-2.3 \pm 1.63)$. No correlations were statistically significant $(\mathrm{p}>0.05)$ (Table 3$)$.

\section{Ranks}

In each group studied, DM had the greatest impact on the patients' "freedom to eat". For Poles and Czechs, the second most affected domain was "freedom to drink", and for Slovaks, "feelings about the future". Conversely, the third most affected domain was "feelings about the future" for Poles and Czechs, and "freedom to drink" for Slovaks (Table 4). The least affected domains in all three groups were "living conditions", followed by "people's reaction".

\section{Regression Analyses}

The applied linear regression model was used to verify whether the selected socio-demographic factors (including gender, age, education, residence, marital status, professional activity) or clinical parameters (HbAlc levels, fasting blood glucose, BMI, duration of DM, complications, treatment used) can affect QoL by lowering the AWI score.

For Polish patients, the linear regression model demonstrated the following significant $(\mathrm{p}<0.05)$ AWI predictors:

- pre-university education: the regression parameter is 1.396 , meaning that pre-university education increases the AWI score by a mean of 1.396 points compared to primary or vocational education;

- past smoking: the regression parameter is -1.459 , meaning that past smoking decreases the AWI score by a mean of 1.459 points compared to never having smoked.

In the Polish group, the $\mathrm{R}^{2}$ coefficient for the model was $29.68 \%$, meaning that variables included in the model account for $29.68 \%$ of variance in AWI scores. The remaining $70.32 \%$ depends on variables not included in the model or random factors (Table 5).

For Czech patients, the linear regression model demonstrated that none of the characteristics analyzed were significant independent predictors of AWI (as all $\mathrm{p}>0.05$ ).

In the Czech group, the $\mathrm{R}^{2}$ coefficient for the model was $9.81 \%$, meaning that variables included in the model account for $9.81 \%$ of variance in AWI scores. The remaining $90.19 \%$ depends on variables not included in the model or random factors (Table 5).

For Slovak patients, the linear regression model demonstrated the following significant $(\mathrm{p}<0.05)$ AWI predictors:

- higher education: the regression parameter is 23.067, meaning that higher education decreases the 
Table I Patient Characteristics by Country

\begin{tabular}{|c|c|c|c|c|c|}
\hline \multicolumn{2}{|l|}{ Parameter } & \multicolumn{3}{|l|}{ Country } & \multirow{2}{*}{$\begin{array}{l}\mathbf{p} \\
\mathrm{p}=0.001 *\end{array}$} \\
\hline & & \multirow{2}{*}{$\begin{array}{l}P L(N=2 \mid 4) \\
61.53 \pm 7.76 \\
62 \\
55-65\end{array}$} & \multirow{2}{*}{$\begin{array}{l}C Z(N=196) \\
59.16 \pm 7 \\
58 \\
54.75-65\end{array}$} & \multirow{2}{*}{$\begin{array}{l}\text { SK }(\mathbf{N}=198) \\
58.8 \pm 7.02 \\
61 \\
52-62\end{array}$} & \\
\hline Age [years] & $\begin{array}{l}\text { Mean } \pm S D \\
\text { Median } \\
\text { Quartiles }\end{array}$ & & & & $\begin{array}{l}\mathrm{P}=0.00 \mathrm{I} * \\
\mathrm{PL}>\mathrm{CZ}, \mathrm{SK}\end{array}$ \\
\hline BMI $\left[\mathrm{kg} / \mathrm{m}^{2}\right]$ & $\begin{array}{l}\text { Mean } \pm \text { SD } \\
\text { Median } \\
\text { Quartiles }\end{array}$ & $\begin{array}{l}26.18 \pm 4.67 \\
25.77 \\
23.45-28.48\end{array}$ & $\begin{array}{l}26.87 \pm 3.79 \\
25.25 \\
23.74-29.06\end{array}$ & $\begin{array}{l}25.28 \pm 2.6 \\
24.39 \\
23.43-28.73\end{array}$ & $\begin{array}{l}\mathrm{P}<0.00 \mathrm{I} * \\
\mathrm{CZ}, \mathrm{PL}>\mathrm{SK}\end{array}$ \\
\hline Diabetes duration [years] & $\begin{array}{l}\text { Mean } \pm \text { SD } \\
\text { Median } \\
\text { Quartiles }\end{array}$ & $\begin{array}{l}5.83 \pm 2.67 \\
6 \\
4-8\end{array}$ & $\begin{array}{l}5.36 \pm 2.4 \\
5 \\
3.75-7\end{array}$ & $\begin{array}{l}6 \pm 2.25 \\
6 \\
4-7\end{array}$ & $\begin{array}{l}\mathrm{P}=0.02 * \\
\mathrm{SK}>\mathrm{CZ}\end{array}$ \\
\hline Glucose fasting $[\mathrm{mg} / \mathrm{dL}]$ & $\begin{array}{l}\text { Mean } \pm \text { SD } \\
\text { Median } \\
\text { Quartiles }\end{array}$ & $\begin{array}{l}154.32 \pm 56.26 \\
135 \\
123-179.5\end{array}$ & $\begin{array}{l}|56.43 \pm 4| .61 \\
140 \\
|2|-189\end{array}$ & $\begin{array}{l}151.09 \pm 38.43 \\
132 \\
121-189\end{array}$ & $\mathrm{p}=0.331$ \\
\hline $\mathrm{HbAlc}[\%]$ & $\begin{array}{l}\text { Mean } \pm S D \\
\text { Median } \\
\text { Quartiles }\end{array}$ & $\begin{array}{l}7.57 \pm 0.9 \\
7.6 \\
6.8-8.1\end{array}$ & $\begin{array}{l}7.89 \pm 1.4 \\
7.2 \\
6.8-8.9\end{array}$ & $\begin{array}{l}7.72 \pm 1.47 \\
7.2 \\
6.8-8.9\end{array}$ & $p=0.224$ \\
\hline Gender & $\begin{array}{l}\text { Female } \\
\text { Male }\end{array}$ & $\begin{array}{l}100(46.73 \%) \\
\text { II4 (53.27\%) }\end{array}$ & $\begin{array}{l}82(41.84 \%) \\
114(58.16 \%)\end{array}$ & $\begin{array}{l}96(48.48 \%) \\
102(51.52 \%)\end{array}$ & $p=0.389$ \\
\hline Education & $\begin{array}{l}\text { Vocational or primary } \\
\text { Pre-university } \\
\text { Higher }\end{array}$ & $\begin{array}{l}95(44.39 \%) \\
84(39.25 \%) \\
35(16.36 \%)\end{array}$ & $\begin{array}{l}86(43.88 \%) \\
92(46.94 \%) \\
18(9.18 \%)\end{array}$ & $\begin{array}{l}87(43.94 \%) \\
101(51.01 \%) \\
10(5.05 \%)\end{array}$ & $p=0.003 *$ \\
\hline Place of residence & $\begin{array}{l}\text { Rural } \\
\text { Urban } \\
\text { Unknown }\end{array}$ & $\begin{array}{l}74(34.58 \%) \\
\text { I } 39(64.95 \%) \\
\text { I }(0.47 \%)\end{array}$ & $\begin{array}{l}88(44.90 \%) \\
108(55.10 \%) \\
0(0.00 \%)\end{array}$ & $\begin{array}{l}123(62.12 \%) \\
75(37.88 \%) \\
0(0.00 \%)\end{array}$ & $\mathrm{p}<0.001 *$ \\
\hline Marital status & $\begin{array}{l}\text { Not in relationship } \\
\text { In relationship } \\
\text { Unknown }\end{array}$ & $\begin{array}{l}68(3 \mid .78 \%) \\
\mid 44(67.29 \%) \\
2(0.93 \%)\end{array}$ & $\begin{array}{l}50(25.5 \mid \%) \\
\mid 46(74.49 \%) \\
0(0.00 \%)\end{array}$ & $\begin{array}{l}54(27.27 \%) \\
\mid 44(72.73 \%) \\
0(0.00 \%)\end{array}$ & $p=0.234$ \\
\hline Professional activity & $\begin{array}{l}\text { Currently working } \\
\text { Not working }\end{array}$ & $\begin{array}{l}64(29.91 \%) \\
150(70.09 \%)\end{array}$ & $\begin{array}{l}96(48.98 \%) \\
100(51.02 \%)\end{array}$ & $\begin{array}{l}75(37.88 \%) \\
123(62.12 \%)\end{array}$ & $\mathrm{p}<0.001 *$ \\
\hline Smoking & $\begin{array}{l}\text { Never } \\
\text { Past } \\
\text { Present } \\
\text { Unknown }\end{array}$ & $\begin{array}{l}84(39.25 \%) \\
49(22.90 \%) \\
79(36.92 \%) \\
2(0.93 \%)\end{array}$ & $\begin{array}{l}10 \mid(5 I .53 \%) \\
40(20.41 \%) \\
55(28.06 \%) \\
0(0.00 \%)\end{array}$ & $\begin{array}{l}134(67.68 \%) \\
34(17.17 \%) \\
30(15.15 \%) \\
0(0.00 \%)\end{array}$ & $\mathrm{p}<0.001 *$ \\
\hline Alcohol & $\begin{array}{l}\text { Drinking } \\
\text { Not drinking }\end{array}$ & $\begin{array}{l}76(35.51 \%) \\
138(64.49 \%)\end{array}$ & $\begin{array}{l}63(32.14 \%) \\
133(67.86 \%)\end{array}$ & $\begin{array}{l}34(17.17 \%) \\
164(82.83 \%)\end{array}$ & $\mathrm{p}<0.001 *$ \\
\hline Comorbidities & $\begin{array}{l}\text { Coronary artery disease } \\
\text { Hypertension } \\
\text { Heart failure } \\
\text { Renal failure } \\
\text { Eye diseases }\end{array}$ & $\begin{array}{l}64(29.91 \%) \\
172(80.37 \%) \\
45(21.03 \%) \\
36(16.82 \%) \\
69(32.24 \%)\end{array}$ & $\begin{array}{l}57(29.08 \%) \\
183(93.37 \%) \\
4 \mid(20.92 \%) \\
34(17.35 \%) \\
7 \mid(36.22 \%)\end{array}$ & $\begin{array}{l}63(31.82 \%) \\
191(96.46 \%) \\
42(21.21 \%) \\
34(17.17 \%) \\
90(45.45 \%)\end{array}$ & $\begin{array}{l}\mathrm{p}=0.781 \\
\mathrm{p}<0.001 * \\
\mathrm{p}=0.921 \\
\mathrm{p}=0.974 \\
\mathrm{p}=0.051\end{array}$ \\
\hline Drugs & $\begin{array}{l}\text { Oral antidiabetic } \\
\text { Insulin } \\
\text { Antihypertensive } \\
\text { Statins }\end{array}$ & $\begin{array}{l}80(37.38 \%) \\
148(69.16 \%) \\
170(79.44 \%) \\
73(34.11 \%)\end{array}$ & $\begin{array}{l}77(39.29 \%) \\
121(61.73 \%) \\
164(83.67 \%) \\
53(27.04 \%)\end{array}$ & $\begin{array}{l}59(29.80 \%) \\
121(61.11 \%) \\
172(86.87 \%) \\
75(37.88 \%)\end{array}$ & $\begin{array}{l}p=0.113 \\
p=0.163 \\
p=0.128 \\
p=0.067\end{array}$ \\
\hline
\end{tabular}

(Continued) 
Table I (Continued).

\begin{tabular}{|l|l|l|l|l|l|}
\hline \multicolumn{2}{|l|}{ Parameter } & \multicolumn{2}{l|}{ Country } & \multicolumn{2}{l|}{ P } \\
\cline { 3 - 6 } \multicolumn{2}{|l|}{} & PL (N=2 14) & CZ (N=196) & SK (N=198) \\
\hline Complications of diabetes & Retinopathy & $77(35.98 \%)$ & $96(48.98 \%)$ & $109(55.05 \%)$ & $P<0.001 *$ \\
& Nephropathy & $30(14.02 \%)$ & $13(6.63 \%)$ & $11(5.56 \%)$ & $P<0.001 *$ \\
& Polyneuropathy & $50(23.36 \%)$ & $48(24.49 \%)$ & $53(26.77 \%)$ & $P=0.958$ \\
& Diabetic foot & $30(14.02 \%)$ & $41(20.92 \%)$ & $42(21.21 \%)$ & $P=0.234$ \\
\hline
\end{tabular}

Notes: $\mathrm{p}-$ Kruskal-Wallis test + post hoc analysis (Dunn test) for quantitative variables, chi-squared or Fisher's exact test for qualitative variables. *Statistically significant $(p<0.05)$.

Table 2 Overall QoL in the Study Group

\begin{tabular}{|c|c|c|c|c|}
\hline \multicolumn{2}{|l|}{ Parameter } & \multicolumn{3}{|l|}{ Country } \\
\hline & & \multirow{4}{*}{$\begin{array}{l}\text { PL (N=2 I 4) } \\
3.4 \mid \pm 0.92 \\
3 \\
3-4\end{array}$} & \multirow{4}{*}{$\begin{array}{l}\mathbf{C Z}(\mathbf{N}=196) \\
3.38 \pm 0.93 \\
3 \\
3-4\end{array}$} & \multirow{4}{*}{$\begin{array}{l}\text { SK }(\mathbf{N}=198) \\
3.47 \pm 0.98 \\
3 \\
3-4\end{array}$} \\
\hline In general, my present quality of life is & Mean $\pm S D$ & & & \\
\hline & Median & & & \\
\hline & Quartiles & & & \\
\hline \multirow[t]{3}{*}{ If I did not have diabetes, my quality of life would be } & Mean $\pm S D$ & $2.65 \pm 0.92$ & $2.57 \pm 0.93$ & $2.6 \pm 0.88$ \\
\hline & Median & 3 & 3 & 3 \\
\hline & Quartiles & $2-3$ & $2-3$ & $2-3$ \\
\hline \multirow[t]{7}{*}{ In general, my present quality of life is } & Excellent $(+3)$ & $\mathrm{I}(0.47 \%)$ & $0(0.00 \%)$ & $0(0.00 \%)$ \\
\hline & Very good $(+2)$ & $28(13.08 \%)$ & $30(15.31 \%)$ & $24(12.12 \%)$ \\
\hline & Good $(+1)$ & $97(45.33 \%)$ & $86(43.88 \%)$ & $90(45.45 \%)$ \\
\hline & Neither good nor bad $(0)$ & $63(29.44 \%)$ & $62(31.63 \%)$ & $59(29.80 \%)$ \\
\hline & $\operatorname{Bad}(-1)$ & $22(10.28 \%)$ & $13(6.63 \%)$ & $19(9.60 \%)$ \\
\hline & Very bad $(-2)$ & $2(0.93 \%)$ & $4(2.04 \%)$ & $3(1.52 \%)$ \\
\hline & Extremely bad $(-3)$ & I (0.47\%) & I (0.5I\%) & $3(1.52 \%)$ \\
\hline \multirow[t]{5}{*}{ If I did not have diabetes, my quality of life would be } & Very much better $(-3)$ & $23(10.75 \%)$ & 27 (13.78\%) & $22(11.11 \%)$ \\
\hline & Much better $(-2)$ & 70 (32.7।\%) & $65(33.16 \%)$ & $66(33.33 \%)$ \\
\hline & A little better $(-1)$ & 81 (37.85\%) & $70(35.71 \%)$ & $80(40.40 \%)$ \\
\hline & The same $(0)$ & $39(18.22 \%)$ & $34(17.35 \%)$ & $30(15.15 \%)$ \\
\hline & Worse (I) & I $(0.47 \%)$ & $0(0.00 \%)$ & $0(0.00 \%)$ \\
\hline
\end{tabular}

Note: $\mathrm{p}$ - Kruskal-Wallis test for quantitative variables, chi-squared or Fisher's exact test for qualitative variables.

AWI score by a mean of 23.067 points compared to primary or vocational education;

- concurrent heart failure: the regression parameter is 29.643, meaning that this comorbidity decreases the AWI score by a mean of 29.643 points.

In the Slovak group, the $\mathrm{R}^{2}$ coefficient for the model was $7.17 \%$, meaning that variables included in the model account for $7.17 \%$ of variance in AWI scores. The remaining $92.83 \%$ depends on variables not included in the model or random factors (Table 5).

\section{Discussion}

Our study provides information on diabetes-related QoL and its evaluation by patients with T2DM in Poland, Slovakia, and the Czech Republic, based on a study using the ADDQoL, a broadly used, DM-specific scale. ${ }^{9-13,19,23}$ In the literature, one can find papers on patients from specific countries, but comparative analyses are scarce.

In recent years, more attention has been paid not only to proper management and ensuring satisfactory adherence, ${ }^{24-26}$ to DM treatment, but also to issues related to self-care and QoL in diabetic patients. ${ }^{27}$ QoL in the physical, psychological, and social domains very often affects adherence and vice versa. The American Diabetes Association guidelines emphasize the need for a "patientcentered" approach to T2DM treatment, so as to preserve or improve the patients' QoL, prevent DM complications and reach blood glucose targets. ${ }^{28}$ 
Table 3 Weighted Impact Scores in the Group

\begin{tabular}{|c|c|c|c|c|}
\hline \multicolumn{2}{|c|}{ Weighted Impact Score } & \multicolumn{3}{|l|}{ Country } \\
\hline & & \multirow{2}{*}{$\begin{array}{l}\text { PL (N=214) } \\
-1.66 \pm 1.72 \\
-2 \\
-2-0\end{array}$} & \multirow{2}{*}{$\begin{array}{l}\mathbf{C Z}(\mathbf{N}=196) \\
-1.68 \pm 1.73 \\
-2 \\
-3-0\end{array}$} & \multirow{2}{*}{$\begin{array}{l}\text { SK }(\mathbf{N}=198) \\
-1.82 \pm 1.76 \\
-2 \\
-3-0\end{array}$} \\
\hline Leisure activities & $\begin{array}{l}\text { Mean } \pm \text { SD } \\
\text { Median } \\
\text { Quartiles }\end{array}$ & & & \\
\hline Working life & $\begin{array}{l}\text { Mean } \pm S D \\
\text { Median } \\
\text { Quartiles }\end{array}$ & $\begin{array}{l}-2.67 \pm 2.85 \\
-2 \\
-4-0\end{array}$ & $\begin{array}{l}-2.68 \pm 2.74 \\
-2 \\
-4-0\end{array}$ & $\begin{array}{l}-2.74 \pm 2.89 \\
-2 \\
-4-0\end{array}$ \\
\hline Journeys & $\begin{array}{l}\text { Mean } \pm \text { SD } \\
\text { Median } \\
\text { Quartiles }\end{array}$ & $\begin{array}{l}-2.13 \pm 2.38 \\
-2 \\
-3-0\end{array}$ & $\begin{array}{l}-2.12 \pm 2.28 \\
-2 \\
-3-0\end{array}$ & $\begin{array}{l}-2.3 I \pm 2.46 \\
-2 \\
-4-0\end{array}$ \\
\hline Holidays & $\begin{array}{l}\text { Mean } \pm \text { SD } \\
\text { Median } \\
\text { Quartiles }\end{array}$ & $\begin{array}{l}-2.42 \pm 2.54 \\
-2 \\
-4-0\end{array}$ & $\begin{array}{l}-2.42 \pm 2.64 \\
-2 \\
-4-0\end{array}$ & $\begin{array}{l}-2.68 \pm 2.74 \\
-2 \\
-4-0\end{array}$ \\
\hline Physical health & $\begin{array}{l}\text { Mean } \pm \text { SD } \\
\text { Median } \\
\text { Quartiles }\end{array}$ & $\begin{array}{l}-2.52 \pm 2.4 \\
-2 \\
-4--1\end{array}$ & $\begin{array}{l}-2.66 \pm 2.55 \\
-2 \\
-4--1\end{array}$ & $\begin{array}{l}-2.62 \pm 2.44 \\
-2 \\
-4--1\end{array}$ \\
\hline Family life & $\begin{array}{l}\text { Mean } \pm \text { SD } \\
\text { Median } \\
\text { Quartiles }\end{array}$ & $\begin{array}{l}-1.92 \pm 2.47 \\
-1 \\
-3-0\end{array}$ & $\begin{array}{l}-2.0 I \pm 2.56 \\
-I \\
-3-0\end{array}$ & $\begin{array}{l}-2.07 \pm 2.5 \\
-2 \\
-3-0\end{array}$ \\
\hline Friendship \& social life & $\begin{array}{l}\text { Mean } \pm \text { SD } \\
\text { Median } \\
\text { Quartiles }\end{array}$ & $\begin{array}{l}-1.91 \pm 2.23 \\
-1 \\
-3-0\end{array}$ & $\begin{array}{l}-1.98 \pm 2.19 \\
-2 \\
-4-0\end{array}$ & $\begin{array}{l}-1.78 \pm 2.21 \\
-1 \\
-3-0\end{array}$ \\
\hline Personal relationship & $\begin{array}{l}\text { Mean } \pm \text { SD } \\
\text { Median } \\
\text { Quartiles }\end{array}$ & $\begin{array}{l}-2.18 \pm 2.69 \\
-1 \\
-4-0\end{array}$ & $\begin{array}{l}-1.82 \pm 2.5 \\
0 \\
-3-0\end{array}$ & $\begin{array}{l}-2.36 \pm 2.79 \\
-2 \\
-4-0\end{array}$ \\
\hline Sex life & $\begin{array}{l}\text { Mean } \pm \text { SD } \\
\text { Median } \\
\text { Quartiles }\end{array}$ & $\begin{array}{l}-2.4 I \pm 2.7 \mid \\
-2 \\
-4-0\end{array}$ & $\begin{array}{l}-2.27 \pm 2.66 \\
-2 \\
-4-0\end{array}$ & $\begin{array}{l}-2.6 \pm 2.7 \\
-2 \\
-4-0\end{array}$ \\
\hline Physical appearance & $\begin{array}{l}\text { Mean } \pm S D \\
\text { Median } \\
\text { Quartiles }\end{array}$ & $\begin{array}{l}-2.34 \pm 2.8 \\
-2 \\
-4-0\end{array}$ & $\begin{array}{l}-2.6 \pm 3.03 \\
-2 \\
-4-0\end{array}$ & $\begin{array}{l}-2.5 \pm 2.99 \\
-2 \\
-4-0\end{array}$ \\
\hline Self-confidence & $\begin{array}{l}\text { Mean } \pm S D \\
\text { Median } \\
\text { Quartiles }\end{array}$ & $\begin{array}{l}-2.25 \pm 2.58 \\
-2 \\
-4-0\end{array}$ & $\begin{array}{l}-2.2 \pm 2.5 \\
-2 \\
-4-0\end{array}$ & $\begin{array}{l}-2.43 \pm 2.67 \\
-2 \\
-4-0\end{array}$ \\
\hline Motivation & $\begin{array}{l}\text { Mean } \pm S D \\
\text { Median } \\
\text { Quartiles }\end{array}$ & $\begin{array}{l}-2.48 \pm 2.39 \\
-2 \\
-4-0\end{array}$ & $\begin{array}{l}-2.4 I \pm 2.3 I \\
-2 \\
-4-0\end{array}$ & $\begin{array}{l}-2.53 \pm 2.33 \\
-2 \\
-4-0\end{array}$ \\
\hline People's reaction & $\begin{array}{l}\text { Mean } \pm \text { SD } \\
\text { Median } \\
\text { Quartiles }\end{array}$ & $\begin{array}{l}-1.49 \pm 2.13 \\
0 \\
-2-0\end{array}$ & $\begin{array}{l}-1.32 \pm 1.98 \\
0 \\
-2-0\end{array}$ & $\begin{array}{l}-1.4 \pm 2.01 \\
0 \\
-2-0\end{array}$ \\
\hline Feelings about future & $\begin{array}{l}\text { Mean } \pm S D \\
\text { Median } \\
\text { Quartiles }\end{array}$ & $\begin{array}{l}-2.83 \pm 2.59 \\
-2 \\
-4--0.75\end{array}$ & $\begin{array}{l}-2.8 I \pm 2.54 \\
-2 \\
-4-0\end{array}$ & $\begin{array}{l}-3.07 \pm 2.53 \\
-3 \\
-4--1\end{array}$ \\
\hline
\end{tabular}

(Continued) 
Table 3 (Continued).

\begin{tabular}{|c|c|c|c|c|}
\hline \multicolumn{2}{|c|}{ Weighted Impact Score } & \multicolumn{3}{|l|}{ Country } \\
\hline & & \multirow{2}{*}{$\begin{array}{l}\text { PL (N=2 I 4) } \\
-2.44 \pm 2.39 \\
-2 \\
-4-0\end{array}$} & \multirow{2}{*}{$\begin{array}{l}\mathbf{C Z}(\mathbf{N}=196) \\
-2.56 \pm 2.4 \\
-2 \\
-4-0\end{array}$} & \multirow{2}{*}{$\begin{array}{l}\text { SK }(\mathbf{N}=198) \\
-2.44 \pm 2.32 \\
-2 \\
-4-0\end{array}$} \\
\hline Financial situation & $\begin{array}{l}\text { Mean } \pm S D \\
\text { Median } \\
\text { Quartiles }\end{array}$ & & & \\
\hline Living conditions & $\begin{array}{l}\text { Mean } \pm S D \\
\text { Median } \\
\text { Quartiles }\end{array}$ & $\begin{array}{l}-1.22 \pm 1.99 \\
0 \\
-2-0\end{array}$ & $\begin{array}{l}-1.29 \pm 2.03 \\
0 \\
-2-0\end{array}$ & $\begin{array}{l}-1.31 \pm 2.02 \\
0 \\
-2-0\end{array}$ \\
\hline Dependence on others & $\begin{array}{l}\text { Mean } \pm S D \\
\text { Median } \\
\text { Quartiles }\end{array}$ & $\begin{array}{l}-1.9 \pm 2.46 \\
-1 \\
-3-0\end{array}$ & $\begin{array}{l}-1.91 \pm 2.39 \\
-1.5 \\
-3-0\end{array}$ & $\begin{array}{l}-1.96 \pm 2.42 \\
-2 \\
-3-0\end{array}$ \\
\hline Freedom to eat & $\begin{array}{l}\text { Mean } \pm S D \\
\text { Median } \\
\text { Quartiles }\end{array}$ & $\begin{array}{l}-4.23 \pm 2.69 \\
-4 \\
-6--2\end{array}$ & $\begin{array}{l}-4.16 \pm 2.49 \\
-4 \\
-6--2\end{array}$ & $\begin{array}{l}-4.19 \pm 2.44 \\
-4 \\
-6--2\end{array}$ \\
\hline Freedom to drink & $\begin{array}{l}\text { Mean } \pm S D \\
\text { Median } \\
\text { Quartiles }\end{array}$ & $\begin{array}{l}-3 \pm 2.57 \\
-2 \\
-4--1\end{array}$ & $\begin{array}{l}-2.96 \pm 2.41 \\
-2 \\
-4--1\end{array}$ & $\begin{array}{l}-2.85 \pm 2.5 \\
-2 \\
-4--1\end{array}$ \\
\hline AWI & $\begin{array}{l}\text { Mean } \pm S D \\
\text { Median } \\
\text { Quartiles }\end{array}$ & $\begin{array}{l}P L(N=214) \\
-2.31 \pm 1.6 \\
-2.03 \\
-3.27--1.05\end{array}$ & $\begin{array}{l}C Z(N=196) \\
-2.3 \pm 1.63 \\
-2.13 \\
-3.39--0.89\end{array}$ & $\begin{array}{l}\text { SK }(N=198) \\
-2.38 \pm 1.64 \\
-2.17 \\
-3.35--1.06\end{array}$ \\
\hline
\end{tabular}

Note: $\mathrm{p}-$ Kruskal-Wallis test.

Table 4 Ranks in the Study Group

\begin{tabular}{|l|l|l|l|}
\hline Ranks & PL & CZ & SK \\
\hline Leisure activities & 17 & 17 & 16 \\
Working life & 4 & 4 & 4 \\
Journeys & 13 & 12 & 13 \\
Holidays & 8 & 8 & 5 \\
Physical health & 5 & 5 & 6 \\
Family life & 14 & 13 & 14 \\
Friendship \& social life & 15 & 14 & 17 \\
Personal relationship & 12 & 16 & 12 \\
Sex life & 9 & 10 & 7 \\
Physical appearance & 10 & 6 & 9 \\
Self-confidence & 11 & 11 & 11 \\
Motivation & 6 & 9 & 8 \\
People's reaction & 18 & 18 & 18 \\
Feelings about future & 3 & 3 & 2 \\
Financial situation & 7 & 7 & 10 \\
Living conditions & 19 & 19 & 19 \\
Dependence on others & 16 & 15 & 15 \\
Freedom to eat & 1 & 1 & 1 \\
Freedom to drink & 2 & 2 & 3 \\
\hline
\end{tabular}

The ADDQoL language versions used in the present study have a good internal consistency, confirmed by the authors, and constitute a reliable instrument for investigating the QoL of patients with T2DM. ${ }^{20,29}$

Approx. $60 \%$ of the studied population of patients with T2DM declared at least a good level of QoL. On the other hand, over $80 \%$ of respondents in each country claimed their QoL would have been better without DM.

In the adult T2DM patients from the Czech Republic, Poland and Slovakia, DM was found to have an adverse impact on all 19 ADDQoL domains.

The effect of T2DM on the weighted impact scores in the ADDQoL differed between particular domains. The most unfavorable weighted impact score was observed in aspects of life such as "freedom to eat", "freedom to drink" and "feelings about the future" in Polish and Czech patients; and "freedom to eat", "feelings about the future", and "freedom to drink" in Slovak patients. The smallest influence of T2DM on the weighted impact score was found in terms of 
Table 5 Linear Regression Results for Each Country

\begin{tabular}{|c|c|c|c|c|c|}
\hline \multicolumn{6}{|l|}{ Poland } \\
\hline \multicolumn{2}{|l|}{ Characteristic } & \multirow{2}{*}{$\begin{array}{l}\text { Parameter } \\
\text { ref. } \\
0.466\end{array}$} & \multicolumn{2}{|l|}{$95 \% \mathrm{Cl}$} & \multirow{2}{*}{$\begin{array}{l}\mathbf{P} \\
0.443\end{array}$} \\
\hline Sex & $\begin{array}{l}\text { Female } \\
\text { Male }\end{array}$ & & -0.717 & 1.649 & \\
\hline Age & [years] & 0.044 & -0.023 & 0.11 & 0.203 \\
\hline BMI & {$\left[\mathrm{kg} / \mathrm{m}^{2}\right]$} & -0.042 & -0.134 & 0.049 & 0.367 \\
\hline Education & $\begin{array}{l}\text { Vocational or primary } \\
\text { Pre-university } \\
\text { Higher }\end{array}$ & $\begin{array}{l}\text { ref. } \\
1.396 \\
0.788\end{array}$ & $\begin{array}{l}0.232 \\
-0.837\end{array}$ & $\begin{array}{l}2.56 I \\
2.4 I 2\end{array}$ & $\begin{array}{l}0.021 * \\
0.345\end{array}$ \\
\hline Place of residence & $\begin{array}{l}\text { Rural } \\
\text { Urban }\end{array}$ & $\begin{array}{l}\text { ref. } \\
-0.948\end{array}$ & -2.119 & 0.222 & 0.117 \\
\hline Marital status & $\begin{array}{l}\text { Not in relationship } \\
\text { In relationship } \\
\text { Unknown }\end{array}$ & $\begin{array}{l}\text { ref. } \\
-0.376 \\
-1.061\end{array}$ & $\begin{array}{l}-1.653 \\
-5.455\end{array}$ & $\begin{array}{l}0.901 \\
3.334\end{array}$ & $\begin{array}{l}0.566 \\
0.638\end{array}$ \\
\hline Professional activity & $\begin{array}{l}\text { Currently working } \\
\text { Not working }\end{array}$ & $\begin{array}{l}\text { ref. } \\
-0.575\end{array}$ & -1.884 & 0.734 & 0.392 \\
\hline Smoking & $\begin{array}{l}\text { Never } \\
\text { Past } \\
\text { Present }\end{array}$ & $\begin{array}{l}\text { ref. } \\
-I .459 \\
-I .144\end{array}$ & $\begin{array}{l}-2.479 \\
-2.34\end{array}$ & $\begin{array}{l}-0.44 \\
0.052\end{array}$ & $\begin{array}{l}0.006 * \\
0.065\end{array}$ \\
\hline Alcohol & $\begin{array}{l}\text { Drinking } \\
\text { Not drinking }\end{array}$ & $\begin{array}{l}\text { ref. } \\
-0.751\end{array}$ & -1.998 & 0.497 & 0.242 \\
\hline Diabetes duration & [years] & -0.308 & -0.628 & 0.012 & 0.063 \\
\hline Coronary artery disease (comorbidities) & $\begin{array}{l}\text { No } \\
\text { Yes }\end{array}$ & $\begin{array}{l}\text { ref. } \\
0.708\end{array}$ & -0.291 & 1.707 & 0.169 \\
\hline Hypertension (comorbidities) & $\begin{array}{l}\text { No } \\
\text { Yes }\end{array}$ & $\begin{array}{l}\text { ref. } \\
0.359\end{array}$ & -1.555 & 2.273 & 0.714 \\
\hline Heart failure (comorbidities) & $\begin{array}{l}\text { No } \\
\text { Yes }\end{array}$ & $\begin{array}{l}\text { ref. } \\
0.548\end{array}$ & -0.85 & 1.945 & 0.445 \\
\hline Renal failure (comorbidities) & $\begin{array}{l}\text { No } \\
\text { Yes }\end{array}$ & $\begin{array}{l}\text { ref. } \\
-1.145\end{array}$ & -2.707 & 0.416 & 0.155 \\
\hline Eye diseases (comorbidities) & $\begin{array}{l}\text { No } \\
\text { Yes }\end{array}$ & $\begin{array}{l}\text { ref. } \\
0.622\end{array}$ & -0.384 & 1.628 & 0.23 \\
\hline Oral antidiabetic & $\begin{array}{l}\text { No } \\
\text { Yes }\end{array}$ & $\begin{array}{l}\text { ref. } \\
0.484\end{array}$ & -0.742 & 1.71 & 0.442 \\
\hline Insulin & $\begin{array}{l}\text { No } \\
\text { Yes }\end{array}$ & $\begin{array}{l}\text { ref. } \\
0.096\end{array}$ & -1.199 & 1.391 & 0.885 \\
\hline Antihypertensive & $\begin{array}{l}\text { Yes } \\
\text { No }\end{array}$ & $\begin{array}{l}\text { ref. } \\
0.058\end{array}$ & -1.663 & 1.779 & 0.947 \\
\hline Statins & $\begin{array}{l}\text { Yes } \\
\text { No }\end{array}$ & $\begin{array}{l}\text { ref. } \\
-0.402\end{array}$ & -1.417 & 0.613 & 0.44 \\
\hline Retinopathy (complications of diabetes) & $\begin{array}{l}\text { No } \\
\text { Yes }\end{array}$ & $\begin{array}{l}\text { ref. } \\
-0.093\end{array}$ & -1.151 & 0.966 & 0.864 \\
\hline
\end{tabular}

(Continued) 
Table 5 (Continued).

\begin{tabular}{|c|c|c|c|c|c|}
\hline Nephropathy (complications of diabetes) & $\begin{array}{l}\text { No } \\
\text { Yes }\end{array}$ & $\begin{array}{l}\text { ref. } \\
1.593\end{array}$ & -0.047 & 3.234 & 0.061 \\
\hline Polyneuropathy (complications of diabetes) & $\begin{array}{l}\text { No } \\
\text { Yes }\end{array}$ & $\begin{array}{l}\text { ref. } \\
-0.017\end{array}$ & -1.17 & 1.135 & 0.976 \\
\hline Diabetic foot & $\begin{array}{l}\text { No } \\
\text { Yes }\end{array}$ & $\begin{array}{l}\text { ref. } \\
0.175\end{array}$ & -1.07 & 1.42 & 0.784 \\
\hline $\begin{array}{l}\text { Glucose fasting } \\
\mathrm{HbAlc}\end{array}$ & $\begin{array}{l}{[\mathrm{mg} / \mathrm{dL}]} \\
{[\%]}\end{array}$ & $\begin{array}{l}-0.002 \\
0.198\end{array}$ & $\begin{array}{l}-0.008 \\
-0.301\end{array}$ & $\begin{array}{l}0.004 \\
0.697\end{array}$ & $\begin{array}{l}0.519 \\
0.439\end{array}$ \\
\hline \multicolumn{6}{|l|}{ Czech Republic } \\
\hline Characteristic & & Parameter & $95 \% \mathrm{Cl}$ & & $\mathbf{p}$ \\
\hline Gender & $\begin{array}{l}\text { Female } \\
\text { Male }\end{array}$ & $\begin{array}{l}\text { ref. } \\
-1.244\end{array}$ & -5.261 & 2.774 & 0.545 \\
\hline $\begin{array}{l}\text { Age } \\
\text { BMI } \\
\text { Education }\end{array}$ & $\begin{array}{l}\text { [years] } \\
{[\mathrm{kg} / \mathrm{m} 2]} \\
\text { Vocational or primary } \\
\text { Pre-university } \\
\text { Higher }\end{array}$ & $\begin{array}{l}-0.121 \\
0.222 \\
\text { ref. } \\
2.938 \\
8.491\end{array}$ & $\begin{array}{l}-0.391 \\
-0.289 \\
-6.213 \\
-23.698\end{array}$ & $\begin{array}{l}0.149 \\
0.734 \\
12.089 \\
40.68\end{array}$ & $\begin{array}{l}0.381 \\
0.396 \\
0.53 \\
0.606\end{array}$ \\
\hline Place of residence & $\begin{array}{l}\text { Rural } \\
\text { Urban }\end{array}$ & $\begin{array}{l}\text { ref. } \\
3.639\end{array}$ & -0.63 & 7.907 & 0.097 \\
\hline Marital status & $\begin{array}{l}\text { Not in relationship } \\
\text { In relationship }\end{array}$ & $\begin{array}{l}\text { ref. } \\
-7.177\end{array}$ & -28.053 & 13.699 & 0.501 \\
\hline Professional activity & $\begin{array}{l}\text { Currently working } \\
\text { Not working }\end{array}$ & $\begin{array}{l}\text { ref. } \\
-1.558\end{array}$ & -26.617 & 23.502 & 0.903 \\
\hline Smoking & $\begin{array}{l}\text { Never } \\
\text { Past } \\
\text { Present }\end{array}$ & $\begin{array}{l}\text { ref. } \\
-4.846 \\
-5.362\end{array}$ & $\begin{array}{l}-17.754 \\
-37.085\end{array}$ & $\begin{array}{l}8.062 \\
26.362\end{array}$ & $\begin{array}{l}0.463 \\
0.741\end{array}$ \\
\hline Alcohol & $\begin{array}{l}\text { Drinking } \\
\text { Not drinking }\end{array}$ & $\begin{array}{l}\text { ref. } \\
6.458\end{array}$ & -13.012 & 25.927 & 0.517 \\
\hline $\begin{array}{l}\text { Diabetes duration } \\
\text { Coronary artery disease }\end{array}$ & $\begin{array}{l}\text { [years] } \\
\text { No } \\
\text { Yes }\end{array}$ & $\begin{array}{l}-0.741 \\
\text { ref. } \\
-4.182\end{array}$ & $\begin{array}{l}-4.937 \\
-12.997\end{array}$ & $\begin{array}{l}3.455 \\
4.633\end{array}$ & $\begin{array}{l}0.73 \\
0.354\end{array}$ \\
\hline Heart failure (comorbidities) & $\begin{array}{l}\text { No } \\
\text { Yes }\end{array}$ & $\begin{array}{l}\text { ref. } \\
-0.311\end{array}$ & -7.124 & 6.503 & 0.929 \\
\hline Eye diseases (comorbidities) & $\begin{array}{l}\text { No } \\
\text { Yes }\end{array}$ & $\begin{array}{l}\text { ref. } \\
-1.269\end{array}$ & -4.31 & 1.771 & 0.414 \\
\hline Oral antidiabetic & $\begin{array}{l}\text { No } \\
\text { Yes }\end{array}$ & $\begin{array}{l}\text { ref. } \\
2.239\end{array}$ & -11.324 & 15.802 & 0.747 \\
\hline Statins & $\begin{array}{l}\text { Yes } \\
\text { No }\end{array}$ & $\begin{array}{l}\text { ref. } \\
-7.663\end{array}$ & -29.26 & 13.933 & 0.488 \\
\hline $\begin{array}{l}\text { Glucose fasting } \\
\text { HbAlc }\end{array}$ & $\begin{array}{l}{[\mathrm{mg} / \mathrm{dL}]} \\
{[\%]}\end{array}$ & $\begin{array}{l}0.047 \\
1.412\end{array}$ & $\begin{array}{l}-0.076 \\
-2.484\end{array}$ & $\begin{array}{l}0.171 \\
5.309\end{array}$ & $\begin{array}{l}0.456 \\
0.478\end{array}$ \\
\hline
\end{tabular}


Table 5 (Continued).

\begin{tabular}{|c|c|c|c|c|c|}
\hline \multicolumn{6}{|l|}{ Slovakia } \\
\hline \multicolumn{2}{|l|}{ Characteristic } & \multirow{2}{*}{$\begin{array}{l}\text { Parameter } \\
\text { ref. } \\
1.419\end{array}$} & \multicolumn{2}{|l|}{$95 \% \mathrm{Cl}$} & \multirow{2}{*}{$\begin{array}{l}\mathbf{p} \\
0.411\end{array}$} \\
\hline Gender & $\begin{array}{l}\text { Female } \\
\text { Male }\end{array}$ & & -1.956 & 4.794 & \\
\hline $\begin{array}{l}\text { Age } \\
\text { BMI } \\
\text { Education }\end{array}$ & $\begin{array}{l}\text { [years] } \\
{[\mathrm{kg} / \mathrm{m} 2]} \\
\text { Vocational or primary } \\
\text { Pre-university } \\
\text { Higher }\end{array}$ & $\begin{array}{l}0.861 \\
-5.83 \\
\text { ref. } \\
-63.45 \\
-23.067\end{array}$ & $\begin{array}{l}-0.055 \\
-|1.87| \\
-126.639 \\
-44.301\end{array}$ & $\begin{array}{l}1.777 \\
0.212 \\
-0.262 \\
-1.832\end{array}$ & $\begin{array}{l}0.067 \\
0.06 \\
0.051 \\
0.035 *\end{array}$ \\
\hline Place of residence & $\begin{array}{l}\text { Rural } \\
\text { Urban }\end{array}$ & $\begin{array}{l}\text { ref. } \\
34.866\end{array}$ & -1.241 & 70.973 & 0.06 \\
\hline Marital status & $\begin{array}{l}\text { Not in relationship } \\
\text { In relationship }\end{array}$ & $\begin{array}{l}\text { ref. } \\
79.749\end{array}$ & 0.492 & 159.007 & 0.05 \\
\hline Smoking & $\begin{array}{l}\text { Never } \\
\text { Past } \\
\text { Present }\end{array}$ & $\begin{array}{l}\text { ref. } \\
61.233 \\
-32.496\end{array}$ & $\begin{array}{l}0.17 \\
-65.581\end{array}$ & $\begin{array}{l}122.296 \\
0.589\end{array}$ & $\begin{array}{l}0.051 \\
0.056\end{array}$ \\
\hline Alcohol & $\begin{array}{l}\text { Drinking } \\
\text { Not drinking }\end{array}$ & $\begin{array}{l}\text { ref. } \\
3.257\end{array}$ & -1.362 & 7.876 & 0.169 \\
\hline Diabetes duration & [years] & -2.555 & -5.112 & 0.003 & 0.052 \\
\hline Coronary artery disease (comorbidities) & $\begin{array}{l}\text { No } \\
\text { Yes }\end{array}$ & $\begin{array}{l}\text { ref. } \\
-40.833\end{array}$ & -81.524 & -0.141 & 0.051 \\
\hline Heart failure (comorbidities) & $\begin{array}{l}\text { No } \\
\text { Yes }\end{array}$ & $\begin{array}{l}\text { ref. } \\
-29.643\end{array}$ & -58.529 & -0.758 & $0.046 *$ \\
\hline
\end{tabular}

Notes: $p-$ multivariate linear regression. $*$ Statistically significant $(p<0.05)$.

"living conditions", "people's reactions" and "leisure activities" for Polish and Czech patients. For Slovak patients, the smallest influence of T2DM on the weighted impact score was found in terms of "living conditions", "people's reactions", and "friendship and social life".

In our study, T2DM had the most severe impact on the "freedom to eat" domain, suggesting that patients are most bothered by dietary restrictions or the need to use special nutrition. This is corroborated by previous studies on the QoL of patients with T2DM, both in Poland ${ }^{29}$ and in other countries: Slovenia, ${ }^{4}$ Greece, ${ }^{10}$ Turkey ${ }^{12}$ and Argentina, ${ }^{4}$ as well as in a cross-sectional study including patients from nine European countries. ${ }^{23}$

In the present study, a strong negative impact of DM on patients' "freedom to drink" was also found. Respondents from all three countries reported "freedom to eat" and "freedom to drink" as the two domains most affected. One could presume that these aspects of diabetes education may be suboptimal and require more attention from medical personnel providing recurrent education to patients with T2DM. These results are undoubtedly contributed to by concerns about hyperglycemia, but also about weight gain or excess blood sugar levels. ${ }^{18}$ Increasing the focus on these aspects could also improve metabolic control outcomes in patients with T2DM.

As the "living conditions" domain was the least affected for all patients in our study, T2DM does not seem to adversely affect a patient's financial standing (even though most respondents were not professionally active) or any other aspects of overall living conditions. Notably, alongside "living conditions", other little-affected domains were "people's reactions" and "friendship and social life", which indicates that diabetic patients in all three countries experience the fewest DM-related issues in their social life. Our results are corroborated by those reported in Slovenia by Turk et $\mathrm{al}^{9}$ in Australia by Donald et $\mathrm{al}^{30}$ in Malaysia by Daher et $\mathrm{al}^{31}$ and in China by Kong et al. ${ }^{13}$ 
A study by Kuznetsov L et al, which included over 1800 patients, indicates a link between overall perceived QoL and long-term $\mathrm{HbA1C}$ outcomes. Patients reporting an adverse impact of DM on QoL had significantly higher HbAlC levels after 5 years. The authors suggest that this link between DM-specific QoL and glycemia should be considered in DM management, especially with regard to diet. ${ }^{32}$

In our study, a linear regression model was used to verify whether the selected socio-demographic factors (including gender, age, education, residence, marital status, professional activity) or clinical parameters (HbAlc levels, fasting blood glucose, BMI, duration of DM, complications, treatment used) can affect QoL by lowering the AWI score. For Polish patients, the linear regression model demonstrated that pre-university education and past smoking are significant independent AWI predictors. This means that patients with pre-university education experience a less negative impact of DM on QoL than those with primary or vocational education. ${ }^{33}$ Similar findings were reported by Flatz A. et al in Switzerland. In turn, the QoL of patients who used to smoke was more affected by DM than in the case of those who had never smoked.

In the Polish group, the $\mathrm{R}^{2}$ coefficient for the model was $29.68 \%$, meaning that variables included in the model account for $29.68 \%$ of variance in AWI scores. The remaining $70.32 \%$ depends on variables not included in the model or random factors.

For Czech patients, the linear regression model demonstrated no significant independent predictors of AWI.

In the Czech group, the $\mathrm{R}^{2}$ coefficient for the model was $9.81 \%$, meaning that variables included in the model account for $9.81 \%$ of variance in AWI scores. The remaining $90.19 \%$ depends on variables not included in the model or random factors.

For Slovak patients, the linear regression model demonstrated that higher education and concurrent heart failure are significant independent AWI predictors. In that group, patients who had completed higher education experienced a much more negative impact of DM on QoL than those with primary or vocational education, which runs completely opposite to what Flatz A. found in a group of Swiss patients. Heart failure as a comorbidity also adversely affects DM-related QoL, reflected by the AWI score. A similar effect was reported by Wexler et $\mathrm{al}^{34}$ who found that both heart failure and microvascular complications are associated with poorer perceived QoL.

In the Slovak group, the $\mathrm{R}^{2}$ coefficient for the model was $7.17 \%$, meaning that variables included in the model account for $7.17 \%$ of variance in AWI scores. The remaining $92.83 \%$ depends on variables not included in the model or random factors.

These findings suggest a need for more in-depth studies in larger and more diverse patient groups.

Remarkably, our study did not demonstrate any association between QoL and DM complications, even though about half of the patients studied did have some complications. The literature data are conflicting. Some studies show that patients with no complications have a better QoL, ${ }^{12,20}$ while others show the opposite, ie, that complications have a significant negative impact. ${ }^{35}$

Similarly, insulin therapy for T2DM was not a predictor of poorer QoL in our study group. The reviewed publications by other authors report divergent findings. In the Spanish PANORAMA study, ${ }^{36}$ as well as a cross-sectional study covering nine European countries, ${ }^{23}$ patients treated with insulin experienced a deterioration of QoL. ${ }^{37}$

Our findings regarding metabolic control and obesity are similar. Though all patients had excessive HbA1C levels and were overweight, neither of these factors was found to be an independent predictor of poorer QoL.

\section{Study Strengths and Limitations}

Our results are consistent with those reported by authors of studies performed in other countries. Our study demonstrates that T2DM represents a growing public health issue, not just in the countries studied here, but also elsewhere, as it adversely affects not only patients' health but also their perceived QoL in all of the aspects analyzed. The findings reported here do not fully reflect all the problems experienced by T2DM patients in the populations that were studied, and therefore further studies seem warranted, including other more accurate clinical characteristics and a more diverse patient group, which would allow for the performance of additional analyses.

A strength of the ADDQoL is that it measures quality of life in specific, potentially relevant areas of people's lives. A consequence, however, is that not all areas are applicable to all respondents. As a result, most respondents did not provide complete data for all ADDQoL domains.

\section{Conclusions}

In summary, our findings demonstrate that T2DM has a negative impact on all aspects of patients' QoL, which is the strongest in terms of the freedom to eat and dietary habits, regardless of the country. Education, past smoking, 
and concurrent heart failure were the only independent predictors of QoL in our study. This suggests a need for further research that would include more variables and a larger number of patients.

\section{Disclosure}

The authors report no conflicts of interest in this work.

\section{References}

1. IDF. Diabetes atlas 9th. Available from: https://www.diabetesatlas. org/upload/resources/material/20200302_133351_IDFATLAS9e-final -web.pdf. Accessed July 20, 2020.

2. Kinra S, Bowen LJ, Lyngdoh T, Prabhakaran D, Reddy KS, Ramakrishnan L. Socio-demographic patterning of non-communicable disease risk factors in rural India: a cross sectional study. BMJ. 2010;341(sep27 1):c4974. doi:10.1136/bmj.c4974

3. Available from: https://www.who.int/healthinfo/survey/whoqolqualityoflife/en/.

4. Pichon-Riviere A, Irazola V, Beratarrechea A, Alcaraz A, Carrara C. Quality of life in type 2 diabetes mellitus patients requiring insulin treatment in buenos aires, argentina: a cross-sectional study. Int $J$ Health Policy Manag. 2015;4(7):475-480. doi:10.15171/ ijhpm.2015.80

5. Sundaram M, Kavookjian J, Patrick JH, Miller LA, Madhavan SS, Scott VG. Quality of life, health status and clinical outcomes in Type 2 diabetes patients. Qual Life Res. 2007;16(2):165-177. doi:10.1007/ s11136-006-9105-0

6. Wee HL, Tan CE, Goh SY, Li SC. Usefulness of the audit of diabetes-dependent quality-of-life (ADDQoL) questionnaire in patients with diabetes in a multi-ethnic Asian country. Pharmacoeconomics. 2006;24(7):673-682. doi:10.2165/00019053-200624070-00006

7. Ashwell SG, Bradley C, Stephens JW, Witthaus E, Home PD. Treatment satisfaction and quality of life with insulin glargine plus insulin lispro compared with NPH insulin plus unmodified human insulin in individuals with type 1 diabetes. Diabetes Care. 2008;31 (6):1112-1117. doi: $10.2337 / \mathrm{dc} 07-1183$

8. Cooke D, O'Hara MC, Beinart N, et al. Linguistic and psychometric validation of the diabetes-specific quality-of-life Scale in U.K English for adults with type 1 diabetes. Diabetes Care. 2013;36 (5):1117-1125. doi: $10.2337 / \mathrm{dc} 12-0076$

9. Turk E, Prevolnik Rupel V, Tapajner A, Leyshon S, Isola A. An audit of diabetes-dependent quality of life (ADDQOL) in older patients with diabetes mellitus type 2 in slovenia. Value Health Reg Issues. 2013;2(2):248-253. doi:10.1016/j.vhri.2013.05.001

10. Papazafiropoulou AK, Bakomitrou F, Trikallinou A, et al. Diabetesdependent quality of life (ADDQOL) and affecting factors in patients with diabetes mellitus type 2 in Greece. BMC Res Notes. 2015;8 (1):786. doi:10.1186/s13104-015-1782-8

11. Lemon SC, Rosal MC, Welch G. Measuring quality of life in low-income, Spanish-speaking Puerto Ricans with type 2 diabetes residing in the mainland U.S. Qual Life Res. 2011;20(9):1507-1511. doi:10.1007/s11136-011-9871-1

12. Ozder A, Sekeroglu M, Eker HH. Quality of life and satisfaction with treatment in subjects with type 2 diabetes: results from primary health care in Turkey. Int J Clin Exp Med. 2014;7(12):5715-5722.

13. Kong D, Ding Y, Zuo X, et al. Adaptation of the audit of diabetes-dependent quality of life questionnaire to people with diabetes in China. Diabetes Res Clin Pract. 2011;94(1):45-52. doi:10.1016/j.diabres.2011.05.026

14. Statistical report: diabetes statistics in Poland and in the world. [in Polish]. Available from: http://cukrzycapolska.pl/cukrzyca/statystyki. Accessed November 3, 2018.
15. Available from: https://www.uzis.cz/sites/default/files/knihovna/nzis rep_2018_K01_A004_diabet_endokrin_2017.pdf. Accessed July 20, 2020.

16. Available from: http://www.nczisk.sk/Documents/aktuality/tlacove spravy/2019/TS_diabetes_12112019.pdf. Accessed July 20, 2020.

17. Bradley C, Todd C, Gorton T, Symonds E, Martin A, Plowright R. The development of an individualized questionnaire measure of perceived impact of diabetes on quality of life: the AD-DQoL. Qual Life Res. 1999;8(1/2):79-91. doi:10.1023/A:1026485130100

18. Bradley C, Speight J. Patient perceptions of diabetes and diabetes therapy: assessing quality of life. Diabetes Metab Res Rev. 2002;18 (Suppl 3):S64-S69. doi:10.1002/dmrr.279

19. Bak E, Marcisz C, Nowak-Kapusta Z, et al. Psychometric properties of the audit of diabetes-dependent quality of life (ADDQoL) in a population-based sample of Polish adults with type 1 and 2 diabetes. Health Qual Life Outcomes. 2018;16(53). doi:10.1186/s12955-0180878-y

20. Holmanová E, Žiaková K. Audit of diabetes-dependent quality of life questionnaire: usefulness in diabetes self-management education in the Slovak population. J Clin Nurs. 2009;18(9):1276-1286. doi:10.1111/j.1365-2702.2008.02602.x

21. Brož J, Janickova Zdarska D, Urbanova J, et al. An international, multicenter, observational survey to evaluate diabetes control in subjects using insulin for the treatment of type 1 and type 2 diabetes mellitus in the Czech Republic and Slovak Republic: study protocol for a cross-sectional survey. Open Access $J$ Clin Trials. 2016;8:13-20. doi:10.2147/OAJCT.S103459

22. R Core Team. R: A Language and Environment for Statistical Computing. Vienna, Austria: R Foundation for Statistical Computing; 2017.

23. Bradley C, Eschwège E, de Pablos-velasco $\mathrm{P}$, et al. Predictors of quality of life and other patient-reported outcomes in the PANORAMA multinational study of people with type 2 diabetes. Diabetes Care. 2018;41(2):267-276. doi:10.2337/dc16-2655

24. Hoogendoorn CJ, Shapira A, Roy JF, Walker EA, Cohen HF, Gonzales JS. Depressive symptom dimensions and medication non-adherence in suboptimally controlled type 2 diabetes. J Diabetes Complications. 2019;33 (3):217-222. doi:10.1016/j.jdiacomp.2018.12.001

25. Jannoo Z, Khan NM. Medication adherence and diabetes self-care activities among patients with type 2 diabetes mellitus. Value Health Reg Issues. 2019;18:30-35. doi:10.1016/j.vhri.2018.06.003

26. Hashimoto K, Urata K, Yoshida A, et al. The relationship between patients' perception of type 2 diabetes and medication adherence: a cross-sectional study in Japan. J Pharm Health Care Sci. 2019;5(2). doi:10.1186/s40780-019-0132-8

27. Sousa VD, Hartman SW, Miller EH, Carroll MA. New measures of diabetes self-care agency, diabetes self-efficacy, and diabetes self-management for insulin-treated individuals with type 2 diabetes. J Clin Nurs. 2009;18(9):1305-1312. doi:10.1111/j.1365-2702.2008.02729.x

28. Guideline of American Diabetes Association. Standards of medical care in diabetes-2015: summary of revisions. Diabetes Care. 2015; (38 Suppl):S4. doi:10.2337/dc15-S003

29. Bąk E, Nowak Z, Dobrzyn-Matusiak D, Marcisz-Dyla E, Marcisz C, Krzemińska S. An assessment of diabetes-dependent quality of life (ADDQoL) in women and men in Poland with type 1 and type 2 diabetes. Ann Agric Environ Med. 2019;26(3):429-438. doi:10.26444/ aaem/99959

30. Donald M, Dower J, Coll JR, Baker P, Mukandi B, Doi SAR. Mental health issues decrease diabetes-specific quality of life independent of glycaemic control and complications: findings from Australia's living with diabetes cohort study. Health Qual Life Outcomes. 2013;11 (1):170. doi:10.1186/1477-7525-11-170

31. Daher AM, AlMashoor SHA, Winn T, van Wouwe J. Performance of the malay audit of diabetes dependent quality of life- 18 and associates of quality of life among patients with type 2 diabetes mellitus from major ethnic groups of Malaysia. PLoS One. 2016;11(10): e0163701. doi:10.1371/journal.pone.0163701 
32. Kuznetsov L, Griffin SJ, Davies MJ, et al. Diabetes-specific quality of life but not health status is independently associated with glycaemic control among patients with type 2 diabetes: a cross-sectional analysis of the ADDITION-Europe trial cohort. Diabetes Res Clin Pract. 2014;104(2):281-287. doi:10.1016/j.diabres.2013.12.029

33. Flatz A, Casillas A, Stringhini S, Zuercher E, Burnand B, Peytremann-Bridevaux I. Association between education and quality of diabetes care in Switzerland. Int J Gen Med. 2015;8:87-92. doi:10.2147/IJGM.S77139

34. Wexler DJ, Grant RW, Wittenberg E, et al. Correlates of health-related quality of life in type 2 diabetes. Diabetologia. 2006;49:1489-1497. doi:10.1007/s00125-006-0249-9
35. Wang HF, Yeh MC. The quality of life of adults with type 2 diabetes in a hospital care clinic in Taiwan. Qual Life Res. 2013;22 (3):577-584. doi:10.1007/s11136-012-0178-7

36. DePablos-Velasco P, Salguero-Chaves E, Mata-Poyo J, DerivasOtero B, García-Sánchez R, Viguera-Ester P. Quality of life and satisfaction with treatment in subjects with type 2 diabetes: results in Spain of the panorama study. Endocrinol Nutr. 2014;61(1):18-26. doi:10.1016/j.endoen.2014.01.011

37. Sundaram M, Kavookjian J, Patrick JH. Health-related quality of life and quality of life in type 2 diabetes: relationships in a cross-sectional study. Patient. 2009;2(2):121-133. doi:10.2165/01312067-2009020 20-00008

\section{Publish your work in this journal}

Diabetes, Metabolic Syndrome and Obesity: Targets and Therapy is an international, peer-reviewed open-access journal committed to the rapid publication of the latest laboratory and clinical findings in the fields of diabetes, metabolic syndrome and obesity research. Original research, review, case reports, hypothesis formation, expert opinion and commentaries are all considered for publication. The manuscript management system is completely online and includes a very quick and fair peer-review system, which is all easy to use. Visit http://www.dovepress.com/testimonials.php to read real quotes from published authors. 